\title{
Comparison of 3D Protocols in Metastatic Breast Cancer Tumors
}

\author{
Erin Stempinski ${ }^{1}$, Jessica Riesterer ${ }^{1}$, Christopher K E Bleck ${ }^{2}$, Claudia Lopez ${ }^{1}$ and Joe Gray ${ }^{1}$ \\ ${ }^{1}$ Oregon Health and Science University, Portland, Oregon, United States, ${ }^{2}$ National Heart Lung and Blood \\ Institute, National Institutes of Health, Bethesda, Maryland, United States
}

Focused Ion Beam-Scanning Electron Microscopy (FIB-SEM) and Serial Block Face-Scanning Electron Microscopy are currently being used in the Serial Measurements of Molecular and Architectural Responses to Therapy (SMMART®) clinical trials at Oregon Health and Science University to understand the spatial relationship between fibroblasts, cancer cells, and the tumor stroma in metastatic cancer by collecting 3D image stacks. We further seek to characterize breast cancer sub-types and metastatic sites via cell morphology, as well as understand the effect of nuclear morphology on cancer development. Cancer tissue provides challenges in ultrastructural preservation due to the density of tissue, the extracellular matrix fibers, and necrotizing cells from anoxic conditions. The current protocol used for 3D EM in the SMMART trial is based on slight modifications to a protocol developed by Hua et al. (Hua, Laserstein, \& Helmstaedter, 2015). This protocol was selected based on the strong ultrastructural contrast, consistent staining throughout the tissue of multiple tissue types, and ability to use for both FIB-SEM and SBF-SEM. However, the protocol requires three days of processing prior to polymerization. This amount of time can be difficult to dedicate in a core facility. We therefore compared the Hua method to a method performed by Bleck et al. (Bleck et al, 2018), which only requires two comparatively short days for processing. The two protocols differ mainly in time for each step, the organic solvent used for dehydration, and some small variations in temperature and resin. Both methods resulted in strong membrane staining, consistent throughout the tissue, which allows for future segmentation of cells and nuclei via machine learning methods. The Bleck method saved 8.14 hours of processing time compared to the Hua method. Light microscopy resin sections of samples processed with the Bleck method stained with toluidine blue show complete infiltration and polymerization of the sample, something that has been a challenge with the Hua method. Under SEM, however, the samples appeared to have texture thought to be related to issues with infiltration and polymerization in a sample-dependent manner, suggesting that this step continues to be an issue regardless of method. Overall, the Bleck method for processing samples for 3D shows promise for preservation and contrast of metastatic breast cancer tissue and offers significant time savings.

\section{References}

Bleck, C.K.E., Kim, Y., Willingham, T.B. et al. Subcellular connectomic analyses of energy networks in striated muscle. Nat Commun 9, 5111 (2018).

Hua, Y., Laserstein, P. \& Helmstaedter, M. Large-volume en-bloc staining for electron microscopy-based connectomics. Nat Commun 6, 7923 (2015). 